2. Filler RM, Tracheomalacia. In: Fallis JC, Filler RM, Lemoine $G$, editors. Pediatric thoracic surgery. New York: Elsevier, 1991:163-71.

3. Vaishnav A, MacKinnon AE. New cervical approach for tracheopexy. Br J Surg 1986;73:441-2.
4. Shoemaker R, Palmer G, Brown JW, King H. Aggressive treatment of acquired phrenic nerve paralysis in infants and children. Ann Thorac Surg 1981;32:251-9.

5. Rogers BM. Pediatric thoracoscopy: Where have we come and what have we learned? Ann Thorac Surg 1993;56:704-7.

\title{
CINE COMPUTED TOMOGRAPHY FOR EVALUATION OF TUMORS INVASIVE TO THE THORACIC AORTA: SEVEN CLINICAL EXPERIENCES
}

Toshiya Ohtsuka, MD, ${ }^{\mathrm{a}}$ Manabu Minami, MD, ${ }^{\mathrm{b}}$ Jun Nakajima, MD, ${ }^{\mathrm{a}}$ Tadasu Kohno, MD, ${ }^{\mathrm{a}}$ Kuniyoshi Yagyu, MD, ${ }^{\mathrm{a}}$ and Akira Furuse, MD, ${ }^{a}$ Tokyo, Japan

Cine computed tomography (CT) has been reported to be useful for evaluating vessel flow or function of the heart. ${ }^{1-5}$ We have applied it for the preoperative evaluation of intrathoracic tumors. Before operating for an intrathoracic tumor that has been found by conventional $\mathrm{CT}$ to be in contact with the aorta, it is important to judge whether the tumor has invaded the aorta; if this is the case, most surgeons will cancel the operation because of

From the Departments of Cardiothoracic Surgery and Radiology, ${ }^{\mathrm{b}}$ Faculty of Medicine, University of Tokyo, Tokyo, Japan.

Received for publication July 13, 1995; accepted for publication Dec. 13, 1995.

Address for reprints: Toshiya Ohtsuka, MD, Department of Cardiothoracic Surgery, University of Tokyo, 7-3-1 Hongo,

Bunkyo-ku, Tokyo 113, Japan.

J Thorac Cardiovasc Surg 1996;112:190-2

Copyright (C) 1996 by Mosby-Year Book, Inc.

$0022-5223 / 96 \$ 5.00+0 \quad \mathbf{1 2 / 5 4 / 7 1 1 6 9}$

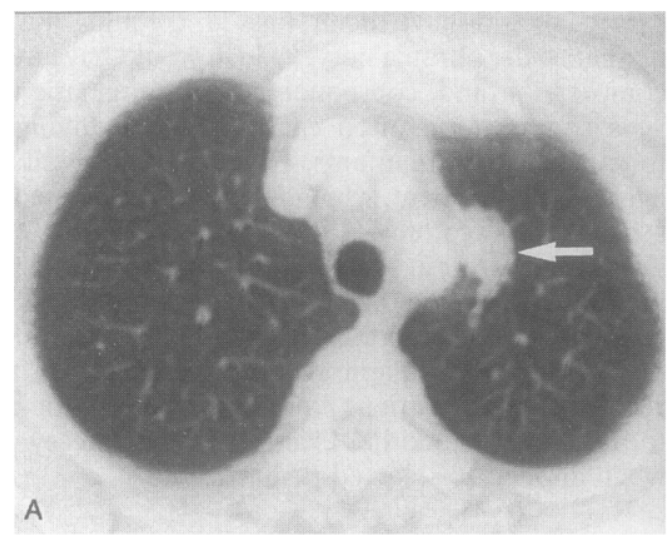

the poor prognosis of a T4 tumor. When a cancer affects the superficial layer of the wall and reveals no sign of invasion by conventional chest $\mathrm{CT}$ or magnetic resonance imaging (MRI), it is virtually impossible to confirm whether malignant adhesion of the tumor exists. To judge preoperatively whether an intrathoracic tumor is invasive to the aorta, we have developed a new method using cine CT. Here we report seven clinical experiences with this new method.

Starting in June 1992, after institutional review board approval and written informed patient consent had been obtained, cine CT was applied for seven consecutive patients with left intrathoracic tumors in contact with the aortic wall. All of the seven tumors, six pulmonary and one thoracoparietal, had been identified previously by conventional chest CT and MRI (Fig. 1). The portions in contact with the seven tumors ranged from the distal arch to the descending aorta. Three pulmonary tumors and a thoracoparietal tumor were adjacent to the distal arch and three lung tumors touched the descending aorta on each side of the mediastinum. Furthermore, one of the lung tumors had invaded the distal arch, which showed obvious deformity and an irregular wall boundary, whereas no

Fig. 1. Standard chest CT scan (A) of tumor 7 (arrow) showing that the tumor was in contact with the aortic wall. B, CT scan of tumor 1. 

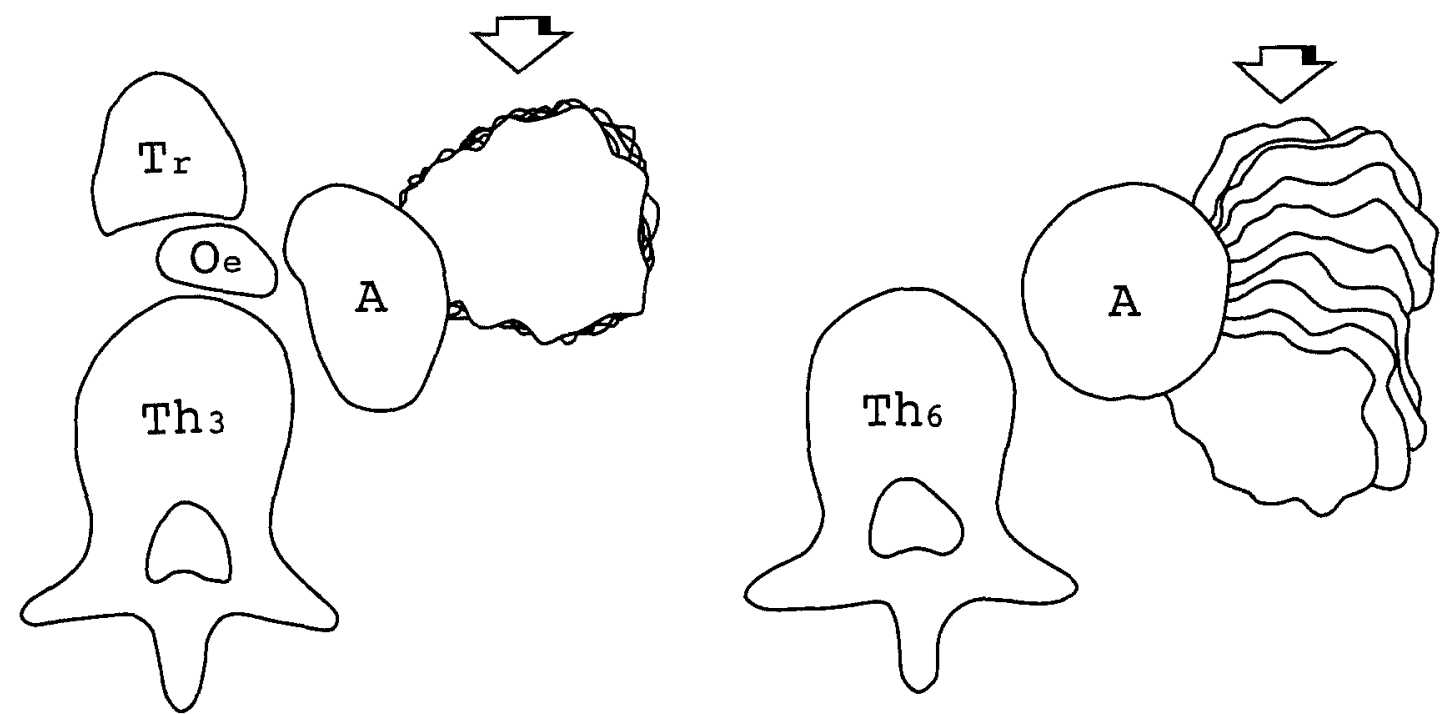

Fig. 2. Traced margins (arrows) of tumors 1 (left) and 7 (right) obtained from ten pictures taken by ultrafast CT showing that the tumors were invasive and noninvasive, respectively, in view of their poor and marked mobility along the aortic wall. $A$, Aorta; $T r$, trachea; Th3,6, third and sixth thoracic vertebrae; $O e$, oesophagus. Fig. 2, left, correlates with Fig. 1, B, and Fig. 2, right, correlates with Fig. 1, A.

Table I. Diagnoses obtained by cine CT and surgical means

\begin{tabular}{ccccc}
\hline & & & \multicolumn{2}{c}{ Diagnosis } \\
\cline { 3 - 4 } Tumor No. & Origin & Adjacent aorta & Cine CT & Operation \\
\hline 1 & Lung & Distal arch & Invasive & Invasive \\
2 & Lung & Distal arch & Invasive & Invasive \\
3 & Chest wall & Distal arch & Noninvasive & Noninvasive \\
4 & Lung & Distal arch & Invasive & Noninvasive \\
5 & Lung & Descending aorta & Invasive & Invasive \\
6 & Lung & Descending aorta & Invasive & Invasive \\
7 & Lung & Descending aorta & Noninvasive & Noninvasive \\
\hline
\end{tabular}

definitive evidence of invasion was obtained for the other six tumors.

Ten pictures are taken at each level by means of ultrafast CT (Imatron C-100, Imatron, San Francisco, Calif.), which can be gated ten times in two different modes during one breath or one heartbeat. A total of eighty pictures are obtained at eight levels in each of the two modes. A cine film is then produced connecting the ten pictures taken at each level. The six pulmonary cancers were examined in the breathing mode, whereas the parietal tumor was examined in the heartbeat mode. Invasion of the aorta was considered to be present if the mobility of tumors along the aorta was found to be poor on observing the motion pictures (Fig. 2). Definitive diagnosis for all of the seven tumors examined was obtained during the operations and was compared with the judgment made from the cine CT.

Cine CT demonstrated that five of the lung tumors were invasive whereas the remaining lung tumor and the tho- racoparietal tumor were not. Thoracoscopic observation revealed a small degree of malignant effusion in one patient and local dissemination of the carcinoma in another with tumors invasive to the distal arch. Surgical treatment for these two patients was canceled because of the advanced nature of the malignant disease. Surgical investigation revealed that two invasive pulmonary cancers, one noninvasive pulmonary cancer in contact with the descending aorta, and one noninvasive parietal tumor that touched the distal arch had been judged correctly by cine CT. However, one pulmonary tumor in contact with the distal arch was proved to have been falsely judged as invasive (Table I). In each of the patients, adhesion was absent except at the site of tumor invasion. The descending aortic walls to be partially resected en bloc with tumors 5 and 6 , involving the lateral wall at the levels of sixth and seventh thoracic vertebrae, respectively, were incised successfully with the aid of a temporary left subclavian-external iliac bypass, then repaired by a patch 
closure technique. Histologically, it was proved in these patients that the cancer affected only the external layer of the aortic wall.

CT was a feasible means of judging whether malignant tumor had invaded the aorta by observing the motion of the tumors along the aortic wall. This method could be used for either purpose-to discriminate T4 tumors from others as a contraindication for resection or to devise a strategy for concomitant resection of the aorta. Provided an invaded portion of the aorta, which is in contact with the immobile surface of a tumor, has been revealed clearly before the operation, a circulatory bypass and prosthesis required for resection and replacement of the wall can be arranged appropriately. In our seven cases, we took advantage of the new imaging modality in this way.

In our series, one tumor in contact with the distal arch was erroneously judged to be invasive. The most likely reason for this misdiagnosis was that the tumor was located on the distal arch near the pulmonary hilus and thus showed barely detectable upward and downward motion during breathing. For the same reason, a noninvasive tumor located on the diaphragm near the pulmonary ligament might be judged invasive by mistake. To avoid such misdiagnoses of pulmonary tumors located on the distal arch or diaphragm, the investigator should use the heartbeat mode as well as the breathing mode for more accurate evaluation, rather than using the breathing mode alone.
It is still difficult to discriminate between invasion and fibrous adhesion. We believe that cine CT should reveal malignant adhesion distinctly, because such invasion shows tougher attachment with less mobility than simple fibrous adhesion. A study including more patients will be required to address this issue.

\section{REFERENCES}

1. Bateman TM, Gray RJ, Whiting JS, Matloff JM, Berman DS, Forrester JS. Cine computed tomographic evaluation of aortocoronary bypass graft patency. J Am Coll Cardiol 1986;8: 693-8.

2. Bateman TM, Gray RJ, Whiting JS, et al. Prospective evaluation of ultrafast cardiac computed tomography for determination of coronary bypass graft. Circulation 1987; 75:1018-24.

3. Froissart M, Archambaud F, Hernigou A, et al. Measurement of global and separate renal blood flow using cine-computed tomography. J Radiol 1994;75:715-21.

4. Garrett JS, Lanzer P, Jaschke W, et al. Measurement of cardiac output by cine computed tomography. Am J Cardiol 1985;56:657-61.

5. Mathru M, Wolfkiel CJ, Jelnin V, et al. Measurement of right ventricular volume in human explanted hearts using ultrafast cine computed tomography. Chest 1994;105:585-8.

\section{APROTININ USE IN PATIENTS WITH DIALYSIS-DEPENDENT RENAL FAILURE UNDERGOING CARDIAC OPERATIONS}

John H. Lemmer, Jr., MD, Mark T. Metzdorff, MD, Albert H. Krause, MD, J. Edward Okies, MD, Thomas A. Molloy, MD, Jonathan G. Hill, MD, William B. Long, MD, Thomas R. Winkler, MD, and U. Scott Page, MD, Portland, Ore.

It is occasionally necessary for patients with chronic renal failure (CRF) who are dependent on dialysis to undergo cardiac operations. Patients with CRF are at increased risk for excessive bleeding and often require substantial blood product transfusions in conjunction with

From Legacy Good Samaritan Hospital, Portland, Ore.

Consulting statistician: Andrea Nadel, $\mathrm{PhD}$, Bayer Corporation, Pharmaceutical Division, West Haven, Conn.

Received for publication Nov. 22, 1995; accepted for publication Jan. 5, 1996.

Address for reprints: John H. Lemmer, Jr., MD, Northwest Surgical Associates, 2226 NW Pettygrove, Portland, OR 97210.

J Thorac Cardiovase Surg 1996;112:192-4

Copyright (c) 1996 by Mosby-Year Book, Inc.

$0022-5223 / 96 \$ 5.00+0 \quad \mathbf{1 2 / 5 4 / 7 1 6 2 5}$ the surgical procedure..$^{1-3}$ Aprotinin, an inhibitor of fibrinolysis with salutary effects on platelet function, decreases bleeding and blood product transfusion requirements when administered during cardiac operations, but published results of aprotinin use in patients with CRF is limited. ${ }^{4-6}$ In this report we describe seven patients dependent on dialysis who received aprotinin during cardiac procedures and compare them with nine patients with CRF who did not.

Methods. Between February 1993 and May 1995, 16 patients with dialysis-dependent CRF underwent cardiac operations done by our group. The patients required dialysis (peritoneal dialysis or hemodialysis) for CRF of various types. All patients underwent dialysis within the 24-hour period before the operation. Standard blood conservation techniques were used in both groups, including tolerance of moderate to severe anemia, use of an intraoperative cell scavenging device, and postoperative 